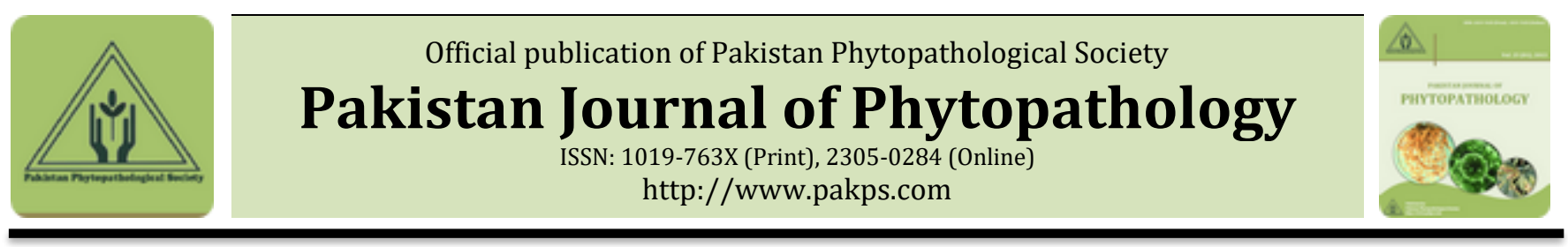

\title{
ANTIFUNGAL ACTIVITY OF DIFFERENT SYSTEMIC FUNGICIDES AGAINST FUSARIUM OXYSPORUM F. SP. LYCOPERSICI ASSOCIATED WITH TOMATO WILT AND EMERGENCE OF RESISTANCE IN THE PATHOGEN
}

\author{
Shazia Akram, Samiya M. Khan, Muhammad F. Khan, Hameed U. Khan, Anam Tariq, Umad U.D. Umar*, Amir Gill \\ Department of Plant Pathology, Faculty of Agricultural Sciences and Technology, \\ Bahauddin Zakariya University Multan-60000, Pakistan.
}

\begin{abstract}
A B S T R A C T
Fusarium wilt in tomato (Lycopersicon esculentum L.) caused by Fusarium oxysporum f. sp. lycopersici. Pathogen is notorious because of its adverse effect on plant growth with causing significant yields losses. Further complication is developing by raising issue of resistance in F. oxysporum $\mathrm{f}$. sp. lycopersici particularly in developing countries due to injudicious fungicides applications in the field of tomato plant infected by this pathogen. F. oxysporum f. sp. lycopersici was isolated from collected infected samples from tomato field in Multan, southern Punjab on Potato dextrose medium by adopting poisoned food technique. Antifungal activity of different systemic fungicides viz. Pyrimethanil, Fludioxonil, Benlate, Bromuconazole, Fosetyl-Al, Flumorph, Prochloraz, Myclobutanil, Epoxiconazole, Strobilurin, carbendazim, Fentin hydroxide, Streptomycin, Tebuconazole, Iprobenfos and azoxystrobin were tested against $F$. oxysporum f. sp. lycopersici in vitro by using poisoned food technique. All tested fungicides suppressed fungal mycelial growth with significantly high or low percent inhibition ranging from 1.48 to $85.92 \%$. Among 16 tested fungicides, different concentrations of Bloom, Prochloraz and Bromuconazole significantly inhibited fungal growth ranging from 75 to $85.92 \%$. Carbendazim and epic reside in the bottom with reference to their efficacy in suppression of $F$. oxysporum f. sp. lycopersici. The results of present research expressed that isolates of Fusarium oxysporum f. sp. lycopersici develops resistance with time which causes reduced efficacy of fungicides as compared to previously published data. The results provided information about fungicides application and selection for the management of holistic disease in tomato crop in Pakistan.
\end{abstract}

Keywords: Tomato, Fungus, Fungicides, Resistance.

\section{INTRODUCTION}

Tomato crop is consumed as a food of significant nutritional value because it is a rich source of minerals and vitamins (Block et al., 1985; Friedman, 2002). Pakistan had 150 thousand ha area and 57094 tons production of tomato in 2014-2015. Sindh is the highest tomato producing province with tomato grown on an area of 67.46 thousand hectares followed by Balochistan with 31.38 thousand hectare of area while Punjab had

\author{
Submitted: July 07, 2018 \\ Revised: November 07, 2018 \\ Accepted for Publication: November 22, 2018 \\ * Corresponding Author: \\ Email: ummad.umar@bzu.edu.pk \\ (c) 2017 Pak. J. Phytopathol. All rights reserved.
}

18.29 thousand ha under tomato cultivation. Overall an increasing trend is observed in the acreage of tomato crop during the last two decades. Tomato crop faces yield losses due to adverse effects of both biotic and abiotic factors (Abdel-Sayed, 2006). Tomato crop is attacked with wide range of vicious fungal, bacterial, viral and nematode pathogens (Agrios, 2005). Fungal pathogens are considered as major contributor to reduced productions (Stone et al., 2000). The fungal pathogens belonging to family Ascomycota order Hypocreales are most devastating and considered a serious threat to tomato crop (Mandal et al., 2009). The Fusarium oxysporum f. sp. lycopersici belong to family Ascomycota causing major losses 10 to $50 \%$ to tomato crop worldwide and 10 to $90 \%$ annually especially in the 
warmer regions of Pakistan (Lagopodi et al., 2002). It destroys root vascular system and blocks transport system of affected plants resulting in reduced growth or death in severe cases (Haware et al., 1978; Malhotra and Vashistha, 1993). F. oxysporum damages different growth stages of crops by inducing symptoms like yellowing of plant parts, dropping of leaves, wilting or root rot and brown-black discoloration in xylem vessels (Dubey and Singh, 2004). Different management strategies including crop rotation, cultural control, chemical control, resistant cultivars and biological control are employed to overcome this fungus (AboElyousr and Mohamed, 2009). The effectiveness of conventional practices is limited due to saprophytic nature of $F$. oxysporum. Resistant cultivars are regarded as the most appropriate source in tackling this pathogen but their availability at wider scale and long term performance is a serious question (Marquina, 1988). In developing countries, chemical applications are applied as a principal control measure against pathogenic fungi (Rojo et al., 2007). The injudicious Fungicides applications have raised some serious issues like pesticide residues, environmental pollution and development of resistance in target fungus species leading to failure of an antifungal property of the chemical (Waard et al., 1993). In fact, lacking of latest efficacy reports data about fungicides against a particular species leads to injudicious fungicides applications against that target pathogen (Naqvi et al., 2015). Importantly, evaluating the efficacy of different systemic fungicides is essential to develop a control strategy.

Due to unavailability of basic data on efficacy of different systemic fungicides for the control of $F$. oxysporum f. sp. lycopersici in southern Punjab, Pakistan limits our understanding of developing control measures. To fill this major gap, current study was designed to investigate antifungal activity of broad range of systemic fungicides belonging to conventional and new chemical groups against $F$. oxysporum f. sp. lycopersici which arises various questions as a result of increasing resistance in pathogen against the fungicides in Pakistan.

\section{MATERIALS AND METHODS}

Diseases sample collection: Infected tomato plant parts showing typical symptoms of Fusarium wilt were collected from experimental fields of Bahauddin Zakariya University Multan. Samples were preserved in polythene bags and brought in to Mycology laboratory of Department of Plant Pathology, Faculty of Agriculture Sciences and Technology B.Z. University Multan $\left(30.1984^{\circ} \mathrm{N}, 71.4687^{\circ} \mathrm{E}\right)$. Infected parts were used as a source of isolation of the pathogen on artificial growth medium i.e., Potato dextrose agar (PDA) (Chohan et al., 2011). Infected parts were cut into small pieces (4-5 mm long) and were washed under tap water and disinfected with $3 \%$ solution of sodium hypochlorite for two minutes. After disinfection, these pieces were rinsed with sterilized distilled water and dried on filter paper. $10 \mathrm{ml}$ of PDA was poured in sterilized petri dishes of 9 $\mathrm{cm}$ diameter in laminar flow chamber. After solidification of medium, these infected pieces of leaves were placed on PDA at $25 \pm 2^{\circ} \mathrm{C}$ for 4 days in an incubator. To obtain pure culture single spore culture technique was adopted. After incubation, petri plates were examined for fungal identification based on morphological characteristics (Nelson et al., 1983). This purified fungal culture was stored at $4^{\circ} \mathrm{C}$ for further use.

Pathogenicity test: Tomato seed were grown in October in green house of Bahauddin Zakariya University. Tomato seedlings approximately size of 10 inches (Roma) of 30 days were transplanted to autoclaved soil infested with $10 \mathrm{ml}$ of fungal conidial suspension inoculum with a concentration of $10^{6}$ spores $/ \mathrm{ml}$ with the help of (Neubauer) haemocytometer prepared from 7 days old fungal culture in plastic pots of (12 cm diameter) in green house of Bahauddin Zakariya university. After inoculation pots were shifted in greenhouse. Symptoms development was recorded at regular intervals. After symptoms development pathogen was re-isolated from symptomatic parts on artificial medium. Morphological characters of isolated and source culture were compared for full filling requirements of Koch's postulates (Ignjatov et al., 2012).

Fungicides: Antifungal activity of 16 different systemic fungicides (commercial formulations) belonging to different groups was evaluated against $F$. oxysporum $\mathrm{f}$. sp. lycopersici at different doses (Table 1)

Antifungal Activity test: Poison Food technique was used for antifungal assay. Different concentrations including 20,60, 80 and $100 \mu \mathrm{g} \mathrm{ml}^{-1}$ of sixteen fungicides (Table 1) were added to PDA and poured into sterilized petri plates for solidification. An isolated culture of Fusarium oxysporum f. sp. lycopersici was inoculated using sterilized cork borer in petri dishes. An 
experiment was conducted by using complete randomized design (CRD) with three replicates. Inoculated petri plates containing PDA medium free of tested fungicides were maintained as control. These petri plates were incubated at $25^{\circ} \mathrm{C}$ for one week until test fungus gained $9 \mathrm{~cm}$ radial mycelial growth in control treatments. Antifungal activities of various fungicides at different concentrations were examined against radial mycelial fungal pathogen growth. Reduction in growth in percent decrease was calculated by using this formula (Sahi et al., 2012).

$$
\mathrm{PD}=\frac{(\mathrm{C}-\mathrm{T})}{\mathrm{C}} \times 100
$$

Where PD is percent decrease, C is colony growth of Fusarium in control, Tis colony growth of Fusarium treated plate.

\section{STATISTICAL ANALYSIS}

Experiment was conducted in complete randomized design and fungus radial growth data was subjected to analysis of variance (ANOVA) by using Statistix (8.1). All Treatment means compared by using Fisher's least significant differences (LSD) at $(\mathrm{P}=0.05)$. Inhibition of fungus radial growth for all treatments was calculated as percent decrease.

\section{RESULTS AND DISCUSSION}

Antifungal affects of tested fungicides on mycelial growth of $\boldsymbol{F}$. oxysporum f. sp. Lycopersici: Efficacy of sixteen systemic fungicides against Fusarium oxysporum f. sp. lycopersici is shown in (Table $2 \& 3$ ). Our results clearly showed fungicides have potential to reduce fungal pathogen growth which causes wilt disease to a large extent. In Table 2 and 3 shows detailed efficacies of tested fungicides in relation to inhibition of mycelial growth and percent decrease over control treatment of all the tested fungicides calculated statistically. Different concentrations of Bromuconazole showed significant control in comparison to other tested fungicides as it reduced growth of the fungal colony up to 1.83, $2.202 .36,2.80$ $\mathrm{cm}$ at low concentration (LC) and high concentrations (HC) respectively. Result of defeater and wisdom in reduction of mycelial growth of Fusarium pathogen i.e., $(3.76,4.20,3.60,3.33),(3.16,4.40,3.60,4.66) \mathrm{cm}$ were closely matched at different tested concentration respectively as compared to control it was $9 \mathrm{~cm}$. In case of benomyl at LC and $\mathrm{HC}$ concentrations significantly reduced fungal mycelial growth of $3.26,4.73,3,4.66 \mathrm{~cm}$ as compared to control. Fludioxonil and Pyranil on all similar concentrations significantly decreased fungal growth $3.20,3.96,3.20,4.86 \mathrm{~cm}$ while in case of Pyranil inhibition zone of fungal growth 4.30, 5.66, 6.56, 5.90 cm (Table 2).

Fungicides Epic at both LC concentrations reduced fungal mycelial growth 7.96 and $8 \mathrm{~cm}$ as compared to control but HC concentrations were not found significant as compared to control. Picoxystrobin with similar concentrations reduced fungal mycelial growth $8.06,8.36,8.60,8.50 \mathrm{~cm}$ as compared to control. Fungicides among carbendazim and Hiten, Hiten show better result at HC concentrations with inhibition zones of 5.76, $5.03 \mathrm{~cm}$. while mycelial growth of Fusarium pathogen i.e. 7.93, 7.50, 8.86, 8.80 $\mathrm{cm}$ on all lower and high (LC and $\mathrm{HC}$ ) concentrations of carbendazim respectively. Flare was found less effective with reducing mycelial growth and its growth is $7.06,5.53,5.40,4.60 \mathrm{~cm}$ on $20,50,80,100$ $(\mu \mathrm{g} / \mathrm{mL})$ concentrations respectively. In case of Tebuconazole and benedict, there was decreased at LC concentrations in mycelial growth 7.70, 7.56, 7.60, $7.76 \mathrm{~cm}$ while on $\mathrm{HC}$ concentrations reduction in mycelial growth $8.36,8.53,8.70,7.66 \mathrm{~cm}$ respectively. Azoxystrobin with LC concentrations shows more significant result as reduction in colony diameter i.e. $6.33,6.06 \mathrm{~cm}$ while $\mathrm{HC}$ concentrations give less control with mycelial growth $7.60,7.50 \mathrm{~cm}$ as compared to the control.

Percent inhibition of $F$. oxysporum f. sp. lycopersici colony growth was calculated over control at all tested LC and HC concentrations of fungicides. Bloom was found most significant showing decrease of 85.92, $81.10,79.90 \%$ at HC80, LC 50, HC 100 respectively as compared to control as shown in Table 3. Prochloraz at LC50 and Bromuconazole at HC100 showed $2^{\text {nd }}$ significant fungicides with inhibition of $79.62 \%$. Benomyl and Fludioxonil at LC20, HC80 concentrations showed better result with percent inhibition of 63.6, $64.40 \%$ as compared to control. Pyranil at LC 20 and flare at $\mathrm{HC} 100$ concentrations give satisfactory decrease of 52.21, 48.88\%. Carbendazim and epic at HC80 concentration were less significant with percent inhibition of $1.48 \%$ as compared to all tested concentrations of fungicides shown in Table 3 . Antifungal effects of sixteen systemic fungicides on all tested concentrations on fungal mycelial growth and percent inhibition are shown in Figure $1 \& 2$. 
Pak. J. Phytopathol., Vol. 31 (02) 2018. 169-176

DOI: $10.33866 /$ phytopathol.030.02.0458

Table 1. List of systemic fungicides belongs to various chemical group tested against mycelial growth of F.oxysporum f. sp. lycopersici on PDA medium in vitro

\begin{tabular}{|c|c|c|c|c|c|c|c|c|c|c|c|}
\hline $\begin{array}{l}\text { Sr. } \\
\text { No }\end{array}$ & Trade name & A.I & Action & $\begin{array}{c}\text { A.I } \\
\%\end{array}$ & Formulation & $\begin{array}{l}\text { Dose } /(100 \\
\text { L) }\end{array}$ & $\begin{array}{l}\text { FRAC } \\
\text { group }\end{array}$ & Manufacturing & Chemical Family & A.I & Action \\
\hline 1 & Pyranil & Pyrimethanil & Systemic & 40 & SC & $300 \mathrm{ml}$ & 9 & $\begin{array}{l}\text { Yantai Keda } \\
\text { Chemical Co., } \\
\text { Ltd }\end{array}$ & Pyrimidine & Pyrimethanil & Systemic \\
\hline 2 & Medallion & Fludioxonil & Systemic & & $\mathrm{SC}$ & $125 \mathrm{~g} / \mathrm{L}$ & 12 & Syngenta & Phenylpyrrole & Fludioxonil & Systemic \\
\hline 3 & Benomyl & Benlate & Systemic & 50 & WP & $500 \mathrm{~g} / \mathrm{l}$ & B1 & & Benzimidazole & Benlate & Systemic \\
\hline 4 & Chipco & Bromuconazole & Systemic & 20 & SC & $100 \mathrm{~g} / \mathrm{l})$ & & $\begin{array}{l}\text { Bayer Crop } \\
\text { Science }\end{array}$ & Bromuconazole & Bromuconazole & Systemic \\
\hline 5 & Aliette & Fosetyl-Al & Systemic & 80 & WDG & $250 \mathrm{~g}$ & 33 & Syngenta & $\begin{array}{l}\text { Ethyl } \\
\text { phosphonates }\end{array}$ & Fosetyl-Al & Systemic \\
\hline 6 & Defeater & Flumorph & Systemic & 20 & WDG & $250 \mathrm{~g}$ & 33 & $\begin{array}{l}\text { Bayer } \\
\text { CropScience/Kanzo } \\
\text { AG, }\end{array}$ & Morpholine & Flumorph & Systemic \\
\hline 7 & Sporta & Prochloraz & Systemic & & $\mathrm{EC}$ & $20 \mathrm{~mL}$ & 3 & Bayer & Benzimidazole & Prochloraz & Systemic \\
\hline 8 & Bloom & Myclobutanil & Systemic & 25 & $\mathrm{EC}$ & $40 \mathrm{~mL}$ & 3 & $\begin{array}{l}\text { Four Brothers } \\
\text { Biologic AG } \\
\text { Pakistan, }\end{array}$ & Triazoles & Myclobutanil & Systemic \\
\hline 9 & Epic & Epoxiconazole & Systemic & 12.5 & SC & $160 \mathrm{~mL}$ & 3 & $\begin{array}{l}\text { Pak China } \\
\text { Chemicals (Pvt.) } \\
\text { Ltd., }\end{array}$ & Triazoles & Epoxiconazole & Systemic \\
\hline 10 & Picoxystrobin & Strobilurin & Systemic & 25 & SC & $300 \mathrm{~mL}$ & 11 & $\begin{array}{l}\text { Syngenta Pakistan } \\
\text { Limited, }\end{array}$ & Methoxyacrylates & Strobilurin & Systemic \\
\hline 11 & Bavistin & Carbendazim & Systemic & 50 & $\mathrm{SC}$ & $200 \mathrm{~g} / \mathrm{l})$ & 1 & Syngenta & & carbendazim & Systemic \\
\hline 12 & Hiten & $\begin{array}{l}\text { Fentin } \\
\text { hydroxide }\end{array}$ & Systemic & 50 & SC & $250 \mathrm{~mL}$ & 30 & Kanzo AG, & $\begin{array}{l}\text { Tri phenyl tin } \\
\text { compounds }\end{array}$ & $\begin{array}{l}\text { Fentin } \\
\text { hydroxide }\end{array}$ & Systemic \\
\hline 13 & Flare & Streptomycin & Systemic & 72 & SP & $100 \mathrm{~g}$ & 18 & Kanzo AG, & Cyanoimidazole & Streptomycin & Systemic \\
\hline 14 & Folicur & Tebuconazole & Systemic & 6 & $\mathrm{ME}$ & $750 \mathrm{~mL}$ & 3 & $\begin{array}{l}\text { Pak China } \\
\text { Chemicals (Pvt.) } \\
\text { Ltd., }\end{array}$ & Triazoles & Tebuconazole & Systemic \\
\hline 15 & Benedict & Iprobenfos & Systemic & 50 & $\mathrm{EC}$ & $200 \mathrm{~mL}$ & 6 & $\begin{array}{l}\text { R. B. Avari } \\
\text { Enterprises (Pvt.) } \\
\text { Ltd., }\end{array}$ & $\begin{array}{l}\text { Organophosphate } \\
\text { Esters }\end{array}$ & Iprobenfos & Systemic \\
\hline 16 & Heritage & Azoxystrobin & Systemic & 40 & SC & $180 \mathrm{ml}$ & 11 & Syngenta & Strobilurins & azoxystrobin & Systemic \\
\hline
\end{tabular}

A.I: active ingredient, ${ }^{* *}$ WDG: Water -dispersible Granule ${ }^{* * *}$ WP: Wettable Powder ${ }^{* * * *}$ SC: Suspension Concentrate ${ }^{* * * * *}$ EC: Emulsifiable concentrate, ${ }^{* * * * *}$ 
Table 2. Effects of concentrations of different systemic fungicides on mycelia growth of F. oxysporum f. sp. lycopersici on PDA

\begin{tabular}{|c|c|c|c|c|c|c|c|c|}
\hline \multicolumn{9}{|c|}{ Reduction in Fungal mycelial growth (cm \pm Standard .Error) } \\
\hline $\begin{array}{l}\text { Concentration } \\
(\mu \mathrm{g} / \mathrm{mL})\end{array}$ & Pyranil & Fludioxonil & Benomyl & Bromuconazole & Wisdom & Defeater & Prochloraz & Bloom \\
\hline LC20 & $4.30 \pm 0.17 \mathrm{~d}$ & $3.20 \pm 0.16 \mathrm{c}$ & $3.26 \pm 0.22 \mathrm{~b}$ & $2.36 \pm 0.10 \mathrm{c}$ & $3.16 \pm 0.14 \mathrm{c}$ & $3.76 \pm 1.18 \mathrm{bc}$ & $2.63 \pm 0.22 \mathrm{~b}$ & $2.03 \pm 0.12 \mathrm{~b}$ \\
\hline LC50 & $5.66 \pm 0.27 \mathrm{c}$ & $3.96 \pm 0.12 \mathrm{c}$ & $4.73 \pm 0.52 \mathrm{~b}$ & $2.80 \pm 0.09 \mathrm{~b}$ & $4.40 \pm 0.25 \mathrm{~b}$ & $4.20 \pm 0.34 \mathrm{~b}$ & $1.83 \pm 0.22 \mathrm{c}$ & $1.70 \pm 0.17 \mathrm{c}$ \\
\hline HC80 & $6.56 \pm 0.24 \mathrm{~b}$ & $3.20 \pm 0.34 \mathrm{c}$ & $3.00 \pm 0.33 c$ & $2.20 \pm 0.12 \mathrm{c}$ & $3.60 \pm 0.25 \mathrm{c}$ & $3.60 \pm 1.14 \mathrm{bc}$ & $1.90 \pm 0.05 \mathrm{c}$ & $1.27 \pm 0.07 \mathrm{c}$ \\
\hline HC100 & $5.90 \pm 0.22 \mathrm{bc}$ & $4.86 \pm 0.31 b$ & $4.66 \pm 0.17 \mathrm{~b}$ & $1.83 \pm 0.07 \mathrm{~d}$ & $4.66 \pm 0.17 \mathrm{~b}$ & $3.33 \pm 0.14 \mathrm{c}$ & $2.16 \pm 0.03 \mathrm{bc}$ & $1.80 \pm 0.12 \mathrm{~b}$ \\
\hline Control & $9.00 \pm 0.00 \mathrm{a}$ & $9.00 \pm 0.00 \mathrm{a}$ & $9.00 \pm 0.00 \mathrm{a}$ & $9.00 \pm 0.00 \mathrm{a}$ & $9.00 \pm 0.00 \mathrm{a}$ & $9.00 \pm 0.00 \mathrm{a}$ & $9.00 \pm 0.00 \mathrm{a}$ & $9.00 \pm 0.00 \mathrm{a}$ \\
\hline \multicolumn{9}{|c|}{ *LC: Lower concentration, ** HC: High concentration } \\
\hline Concentration & Epic & Picoxystrobin & Carbendazim & Hiten & Flare & Tebuconazole & Benedict & Azoxystrobin \\
\hline LC 20 & $7.96 \pm 0.07 \mathrm{~b}$ & $8.06 \pm 0.10 \mathrm{c}$ & $7.93 \pm 0.10 \mathrm{~b}$ & $6.80 \pm 0.22 b$ & $7.06 \pm 0.10 \mathrm{~b}$ & $7.70 \pm 0.17 \mathrm{c}$ & $7.60 \pm 0.08 \mathrm{~b}$ & $6.33 \pm 0.17 c$ \\
\hline LC50 & $8.00 \pm 0.19 b$ & $8.36 \pm 0.12 \mathrm{bc}$ & $7.50 \pm 0.21 b$ & $6.70 \pm 0.09 \mathrm{~b}$ & $5.53 \pm 0.24 \mathrm{c}$ & $7.56 \pm 0.10 \mathrm{c}$ & $7.76 \pm 0.15 b$ & $6.06 \pm 1.10 \mathrm{c}$ \\
\hline HC80 & $8.86 \pm 0.11 \mathrm{a}$ & $8.60 \pm 0.09 \mathrm{~b}$ & $8.86 \pm 0.11 \mathrm{a}$ & $5.76 \pm 0.07 \mathrm{c}$ & $5.40 \pm 0.19 c$ & $8.36 \pm 0.12 b$ & $8.70 \pm 0.14 \mathrm{a}$ & $7.60 \pm 0.17 b$ \\
\hline HC100 & $8.70 \pm 0.12 \mathrm{a}$ & $8.50 \pm 0.05 b$ & $8.80 \pm 0.16 \mathrm{a}$ & $5.03 \pm 0.07 \mathrm{~d}$ & $4.60 \pm 0.17 \mathrm{~d}$ & $8.53 \pm 0.20 \mathrm{ab}$ & $7.66 \pm 0.14 \mathrm{~b}$ & $7.50 \pm 0.24 \mathrm{~b}$ \\
\hline Control & $9.00 \pm 0.00 \mathrm{a}$ & $9.00 \pm 0.00 \mathrm{a}$ & $9.00 \pm 0.00 \mathrm{a}$ & $9.00 \pm 0.00 \mathrm{a}$ & $9.00 \pm 0.00 \mathrm{a}$ & $9.00 \pm 0.00 \mathrm{a}$ & $9.00 \pm 0.00 \mathrm{a}$ & $9.00 \pm 0.00 \mathrm{a}$ \\
\hline
\end{tabular}

*LC: Lower concentration, ${ }^{* *}$ HC: High concentration

Means followed by the same letters in each column are not statistically different $(\mathrm{P} \leq 0.05)$

Table 3. Percentage decrease in fungal growth of F. oxysporum f. sp. lycopersici on PDA mixed with systemic fungicides at different concentrations.

\begin{tabular}{|c|c|c|c|c|c|c|c|c|}
\hline \multicolumn{9}{|c|}{$\begin{array}{l}\text { Decrease in Fungal mycelial growth } \\
\text { ( } \% \pm \text { Standard. Error) }\end{array}$} \\
\hline $\begin{array}{l}\text { Concentration } \\
(\mu \mathrm{g} / \mathrm{mL})\end{array}$ & Pyranil & Fludioxonil & Benomyl & Bromuconazole & Wisdom & Defeater & Prochloraz & Bloom \\
\hline LC 20 & $52.21 \pm 1.89 \mathrm{a}$ & $64.40 \pm 1.81 \mathrm{a}$ & $63.69 \pm 2.42 \mathrm{a}$ & $73.70 \pm 1.09 \mathrm{~b}$ & $64.81 \pm 1.51 \mathrm{a}$ & $58.14 \pm 1.98 \mathrm{ab}$ & $70.73 \pm 2.48 b$ & $77.40 \pm 1.32 \mathrm{~b}$ \\
\hline LC50 & $37.04 \pm 3.02 \mathrm{~b}$ & $55.92 \pm .1 .32 \mathrm{a}$ & $47.40 \pm 5.77 \mathrm{~b}$ & $68.88 \pm 1.05 \mathrm{c}$ & $51.18 \pm 2.77 \mathrm{~b}$ & $53.33 \pm 3.78 \mathrm{~b}$ & $79.62 \pm 2.48 \mathrm{a}$ & $81.10 \pm 1.89 \mathrm{ab}$ \\
\hline HC80 & $27.03 \pm 2.69 \mathrm{c}$ & $64.44 \pm 3.78 \mathrm{a}$ & $66.66 \pm 3.67 \mathrm{a}$ & $75.55 \pm 1.39 \mathrm{~b}$ & $59.99 \pm 2.77 \mathrm{a}$ & $59.99 \pm 2.77 \mathrm{ab}$ & $78.88 \pm 0.52 \mathrm{a}$ & $85.92 \pm 0.80 \mathrm{a}$ \\
\hline HC100 & $34.44 \pm 2.40 \mathrm{bc}$ & $45.92 \pm 3.49 \mathrm{~b}$ & $48.14 \pm 1.84 \mathrm{~b}$ & $79.62 \pm 0.80 \mathrm{a}$ & $48.14 \pm 1.84 \mathrm{~b}$ & $62.96 \pm 1.60 \mathrm{a}$ & $75.92 \pm 0.30 \mathrm{ab}$ & $79.90 \pm 1.39 \mathrm{~b}$ \\
\hline Control & $0.00 \pm 0.00 \mathrm{~d}$ & $0.00 \pm 0.00 \mathrm{c}$ & $0.00 \pm 0.00 \mathrm{~b}$ & $0.00 \pm 0.00 \mathrm{~d}$ & $0.00 \pm 0.00 \mathrm{c}$ & $0.00 \pm 0.00 \mathrm{c}$ & $0.00 \pm 0.00 \mathrm{c}$ & $0.00 \pm 0.00 \mathrm{c}$ \\
\hline \multicolumn{9}{|c|}{${ }^{*}$ LC: Lower concentration, ${ }^{* *}$ HC: High concentration } \\
\hline Concentration & Epic & Picoxystrobin & carbendazim & Hiten & Flare & Tebuconazole & Benedict & Azoxystrobin \\
\hline LC 20 & $11.80 \pm 0.80 \mathrm{a}$ & $10.36 \pm 1.09 \mathrm{a}$ & $11.85 \pm 1.09 \mathrm{a}$ & $24.43 \pm 2.40 \mathrm{c}$ & $21.48 \pm 1.09 \mathrm{c}$ & $14.44 \pm 1.89 \mathrm{a}$ & $15.55 \pm 0.91 \mathrm{a}$ & $29.63 \pm 0.17 \mathrm{a}$ \\
\hline LC50 & $11.10 \pm 2.10 \mathrm{a}$ & $7.036 \pm 1.32 \mathrm{ab}$ & $15.92 \pm 2.36 \mathrm{a}$ & $25.55 \pm 1.05 \mathrm{c}$ & $38.52 \pm 2.64 \mathrm{~b}$ & $15.92 \pm 1.09 \mathrm{a}$ & $13.70 \pm 1.68 \mathrm{a}$ & $32.59 \pm 1.09 \mathrm{a}$ \\
\hline HC80 & $1.480 \pm 1.21 \mathrm{~b}$ & $4.40 \pm 1.05 \mathrm{~b}$ & $1.48 \pm 1.21 \mathrm{~b}$ & $35.92 \pm 0.80 \mathrm{~b}$ & $39.99 \pm 2.10 \mathrm{~b}$ & $7.03 \pm 1.32 \mathrm{~b}$ & $3.33 \pm 1.57 \mathrm{~b}$ & $15.55 \pm 1.89 \mathrm{~b}$ \\
\hline HC100 & $3.330 \pm 1.39 \mathrm{~b}$ & $5.55 \pm 0.52 \mathrm{~b}$ & $2.22 \pm 1.81 \mathrm{~b}$ & $44.07 \pm 0.80 \mathrm{a}$ & $48.88 \pm 1.89 \mathrm{a}$ & $5.18 \pm 2.18 \mathrm{bc}$ & $14.81 \pm 1.60 \mathrm{a}$ & $16.66 \pm 2.62 \mathrm{~b}$ \\
\hline Control & $0.00 \pm 0.00 \mathrm{~b}$ & $0.00 \pm 0.00 \mathrm{c}$ & $0.00 \pm 0.00 \mathrm{~b}$ & $0.00 \pm 0.00 \mathrm{~d}$ & $0.00 \pm 0.00 \mathrm{~d}$ & $0.00 \pm 0.00 \mathrm{c}$ & $0.00 \pm 0.00 \mathrm{~b}$ & $0.00 \pm 0.00 \mathrm{c}$ \\
\hline
\end{tabular}

Means followed by the same letters in each column are not statistically different $(\mathrm{P} \leq 0.05)$ 
Figure $1 \& 2$ In-vitro antifungal activity of systemic Fungicides at different fungicides against Fusarium oxysporum $\mathrm{f}$. sp. lycopersici

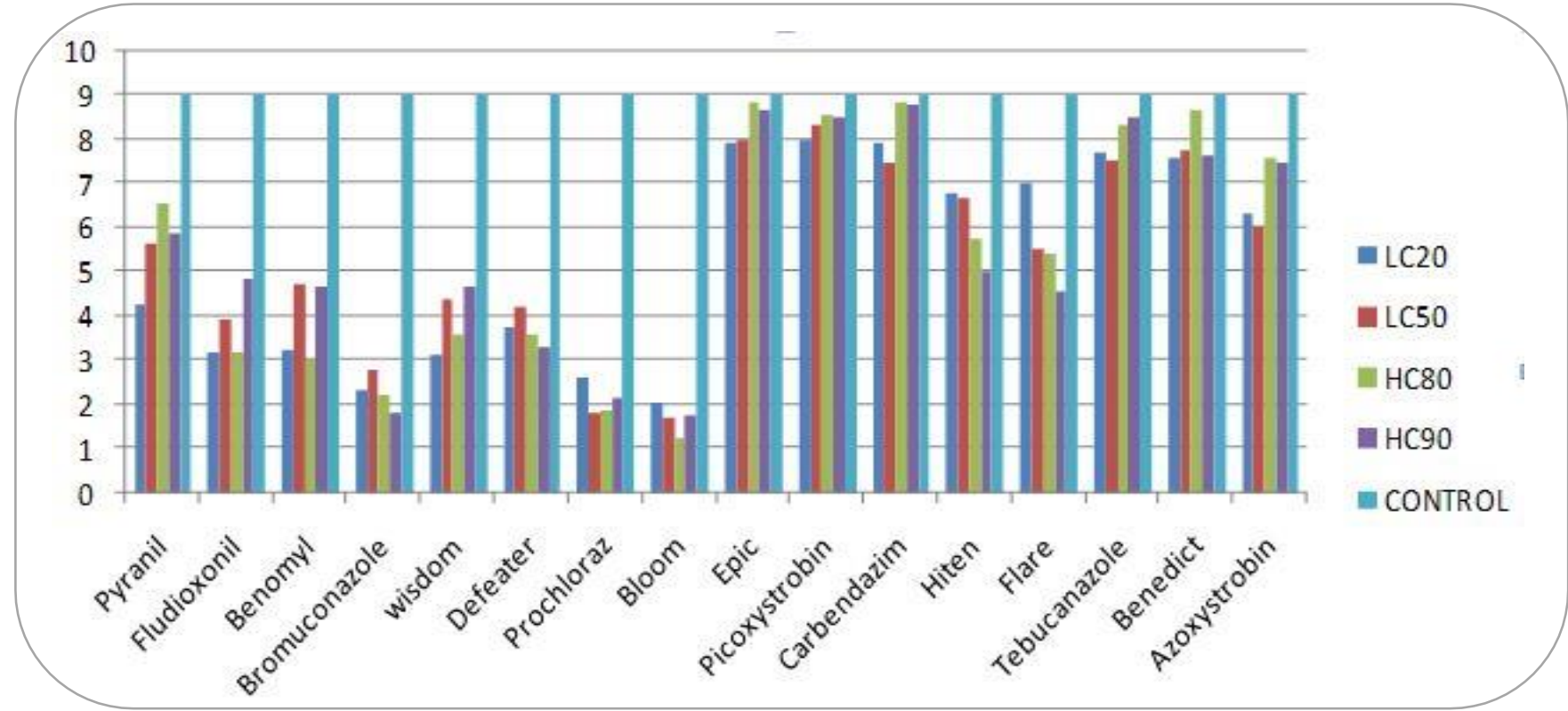

Figure 1. Reduction in colony diameter of Fusarium oxysporum f. sp. lycopersici at different concentrations of fungicides.

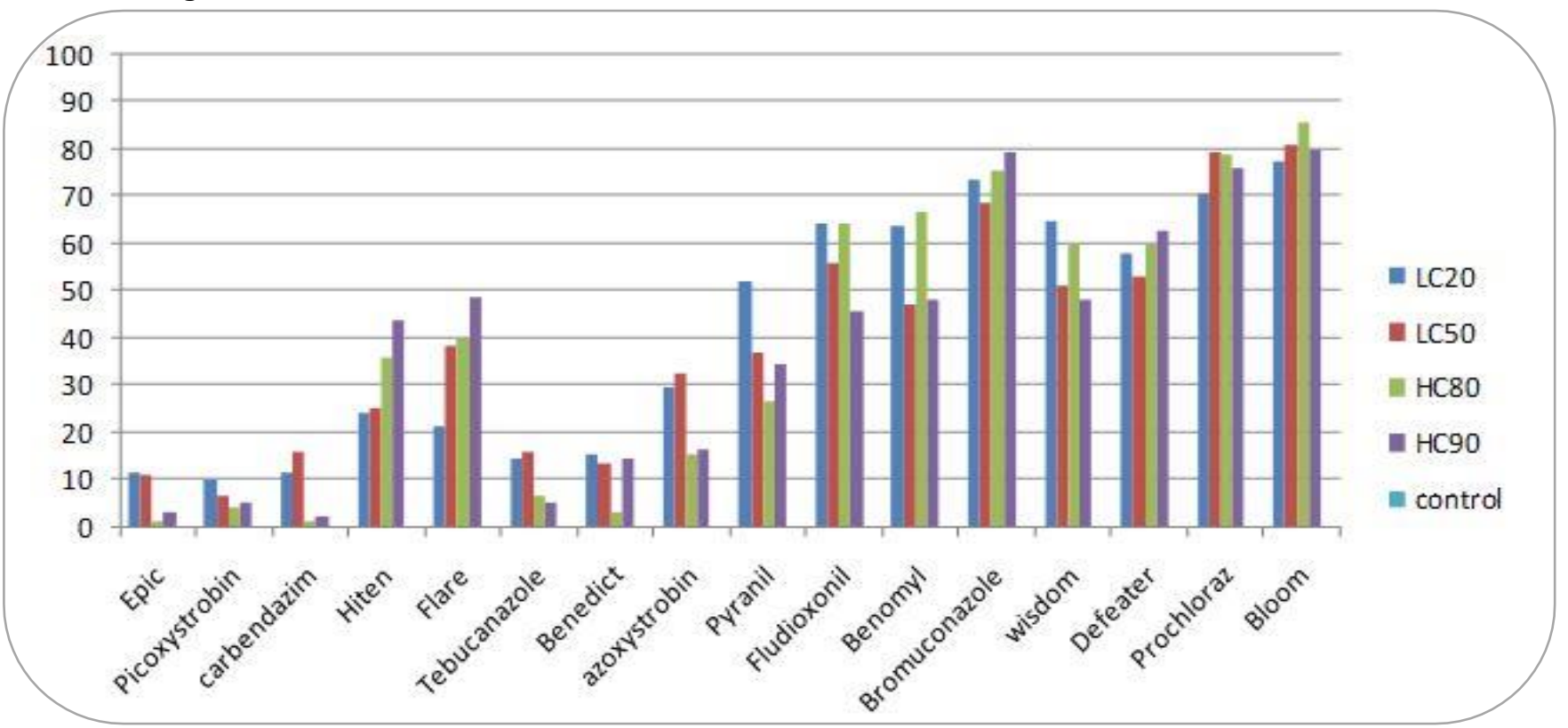

Figure 2. Percent inhibition on different concentrations of fungicides against Fusarium oxysporum f. sp. Lycopersici.

Evaluation of different systemic fungicides for selecting most efficient compounds is main step for pathogen control. Our result showed Bloom, Prochlorazol, Bromuconazole, defeater sand wisdom was most affective in vitro among all tested 16 systemic fungicides by suppressing 75 to $85 \%$ fungal mycelial growth (Amini and Sidovich, 2010; Khan, 2013).The systemic fungicides Benomyl, Pyranil belong to chemical family benzimidazole and Pyrimidine had better curative and preventive effects on Fusarium tomato wilt(Allen et al., 2004).Azoxystrobin was found less effective as fallowed by Fludioxonil (Amini and Sidovich, 2010). Tebuconazole bloom and Epic belong to triazole group caused structural destruction in $F$. oxysporum f. $\mathrm{sp}$. lycopersici by unavailability of ergo sterol to fungi that lead to suppression in growth (Han et al., 2006). 
Tebucanazole and Epic both fungicides significantly less effective as compared to bloom (Khan, 2013).

Antifungal property of Carbendazim, Azoxystrobin and Picoxystrobin may be lost due to resistant development in fungal isolates by heavy pressure created by fungicides application (Pappas, 1997; Reimann and Deising, 2005). Benedict, Hiten and Flare fungicides on different concentrations showed less percent inhibition in fungal mycelial growth as compared to previous work (Akhtar et al., 2014).

Our experiment result showed tested fungicides slowly losing their antifungal affect for controlling wilt disease of tomato crops due to pathogen sensitivity reduction. Various literature stated resistance development problem in Fusarium oxysporum f. sp. lycopersici to fungicides can be solved by formulating new fungicides with advance chemistries to avoid a variety of resistant fungal isolates for longer period of time.

Our result demonstrates that farmers must be aware of significance of rotation practices to dispirit the selection of single chemistry fungicides which significantly lead to resistance development in target pathogens. It is first work in Pakistan on evolution of resistance in $F$. oxysporum f. sp. lycopersici due to malpractice application of systemic fungicides application in tomato groves. Our result reinforcing the fungicides resistance is severe problem of tomato crop in Pakistan.

Our finding is questioning on sustainability of today management strategies against $F$. oxysporum f. sp. lycopersici which exclusively depends on fungicides application and there is need to develop new control strategies which satisfactory control to disease and pathogen.

\section{REFERENCES}

Abdel-Sayed, M. 2006. Pathological, physiological and molecular variations among isolates of Alternaria solani the causal of tomato early blight disease. $\mathrm{Ph}$. D. Thesis, Fac. Agric. Cairo Univ.

Abo-Elyousr, K. A. M. and H. M. Mohamed. 2009. Note Biological Control of Fusarium Wilt in Tomato by Plant Growth-Promoting Yeasts and Rhizobacteria. The Plant Pathology Journal, 25: 199-204.

Agrios, G. N. 2005. Introduction to plant pathology. Elsevier Academic Press Publication.

Akhtar, N., A. Shoaib and A. A. Nafisa. 2014. Pakistan Journal of Phytopathology. Pakistan Journal of Phytopathology, 26: 101-106.
Amini, J. and D. Sidovich. 2010. The Effects of Fungicides on Fusarium Oxysporum F. SP. Lycopersici Associated with Fusarium Wilt of Tomato. Journal of Plant Protection Research, 50.

Block, G., C. M. Dresser, A. M. Hartman and M. D. Carroll. 1985. Nutrient sources in the american diet: quantitative data from the nhanes ii survey. American Journal of Epidemiology, 122: 13-26.

Chohan, S., R. Atiq, M. A. Mehmood, S. Naz, B. Siddique and G. Yasmin. 2011. Efficacy of few plant extracts against Fusarium oxysporum f. sp. gladioli, the cause of corm rot of gladiolus. Journal of Medicinal Plants Research, 5: 3887-3890.

Dubey, S. and B. Singh. 2004. Reaction of chickpea genotypes against Fusarium oxysporum f. sp. ciceri causing vascular wilt. Indian Phytopathology, 57: 233-233.

Friedman, M. 2002. Tomato Glycoalkaloids: Role in the Plant and in the Diet. Journal of Agricultural and Food Chemistry, 50: 5751-5780.

Han, Q., Z. Kang, H. Buchenauer, L. Huang and J. Zhao. 2006. Cytological and immunocytochemical studies on the effects of the fungicide tebuconazole on the interaction of wheat with stripe rust. Journal of Plant Pathology: 263-271.

Haware, M. P. 1978. Eradication of Fusarium oxysporum f. sp. ciceri Transmitted in Chickpea Seed. Phytopathology, 68: 1364.

Ignjatov, M., D. Milosevic, Z. Nikolic, J. GvozdanovicVarga, D. Jovicic and G. Zdjelar. 2012. Fusarium oxysporum as causal agent of tomato wilt and fruit rot. Pesticidi i fitomedicina, 27: 25-31.

Khan, A. 2013. In vitro chemical control of Fusarium oxysporum f. sp. lycopersici. Mycophytopathology, 10.

Lagopodi, A. L., A. F. J. Ram, G. E. M. Lamers, P. J. Punt, C. A. M. J. J. Van den Hondel, B. J. J. Lugtenberg and G. V. Bloemberg. 2002. Novel Aspects of Tomato Root Colonization and Infection by Fusarium oxysporum f. sp. radicis-lycopersici Revealed by Confocal Laser Scanning Microscopic Analysis Using the Green Fluorescent Protein as a Marker. Molecular Plant-Microbe Interactions, 15: 172179.

Malhotra, S. and R. Vashistha. 1993. Genetics of resistance to Fusarium wilt race 1 in currant tomato Lycopersicon pimpinellifolium. Indian Journal of Agricultural Sciences, 63: 246-247. 
Mandal, S., N. Mallick and A. Mitra. 2009. Salicylic acidinduced resistance to Fusarium oxysporum f. sp. lycopersici in tomato. Plant Physiology and Biochemistry, 47: 642-649.

Naqvi, S. A. H., M. R. Latif, S. A. Khan, M. T. Malik and S. Freed. 2015. Emerging resistance against different fungicides in Lasiodiplodia theobromae, the cause of mango dieback in Pakistan. Archives of Biological Sciences, 67: 241-249.

Nelson, P. E., T. A. Toussoun and W. Marasas. 1983. Fusarium species: an illustrated manual for identification.

Pappas, A. C. 1997. Evolution of fungicide resistance in Botrytis cinerea in protected crops in Greece. Crop Protection, 16: 257-263.

Reimann, S. and H. B. Deising. 2005. Inhibition of Efflux Transporter-Mediated Fungicide Resistance in Pyrenophora tritici-repentis by a Derivative of 4'Hydroxyflavone and Enhancement of Fungicide Activity. Applied and Environmental Microbiology, 71: 3269-3275.

Rojo, F. G., M. M. Reynoso, M. Ferez, S. N. Chulze and A. M.
Torres. 2007. Biological control by Trichoderma species of Fusarium solani causing peanut brown root rot under field conditions. Crop Protection, 26: 549-555.

Sahi, S., A. Habib, M. Ghazanfar and A. Badar. 2012. In vitro evaluation of different fungicides and plant extracts against Botryodiplodia theobromae, the causal agent of quick decline of mango. Pakistan Journal of Phytopathology, 24: 137-142.

Stone, J. K., C. W. Bacon and J. F. White Jr. 2000. An overview of endophytic microbes: endophytism defined, Microbial endophytes. CRC Press, pp. 1744.

Tello-Marquina, J. and A. Lacasa. 1988. Evolution of races among Fusarium oxysporum f. sp. lycopersici. De Sanidad Vegetal Plagas, 14: 335-341.

Waard, M. A., S. G. Georgopoulos, D. W. Hollomon, H. Ishii, P. Leroux, N. N. Ragsdale and F. J. Schwinn. 1993. Chemical Control of Plant Diseases: Problems and Prospects. Annual Review of Phytopathology, 31: 403-421. 